sociation of the glucagon binding site in a "solubilized" adenylate cyclase of myocardial origin. Glucagon binding could apparently be separated from adenylate cyclase activity by prior incubation with the hormone. The adenylate cyclase in the detergent used has been previously reported to be glucagon insensitive, however, so the authors are to be commended for their restraint in referring only to a glucagon binding site, and not to a receptor. The use of SDS-acrylamide gels to determine the molecular weight of the complex, using the ${ }^{125}$ I-glucagon as a marker, depends on the incomplete denaturation of the binding component, and the resulting value should perhaps be regarded with caution.

Because of the difficulties of solubilizing hormone-sensitive adenylate cyclases, and the general lack of sensitivity following the removal of lipids, there has been considerable speculation about an obligatory role for lipids in the macromolecular complex. Kreiner et al. (Proc. natn. Acad. Sci., U.S.A., 79, 1785 ; 1973) have detected a phenomenon which may be dependent on the lipid components - a temperature dependence of the energy of activation of the glucagon-stimulated hepatic adenylate cyclase. The effect is not seen in the fluoride or prostaglandin $\mathrm{E}_{1}$ stimulation. As the authors note, although the mole fraction of cholesterol is high (about 0.3), indicating a small bulk phase transition which may be at a temperature rather different from that observed, it is quite possible that the observed transition is restricted to the environs of the enzyme, where the lipid composition could be markedly different from the bulk. A proper demonstration of protein or lipid as the temperaturesensitive component must presumably await the development of better techniques for reconstitution studies.

\section{PSYCHIATRY \\ Biochemical Psychoses}

from a Correspondent

Most people with untreated phenylketonuria (PKU) are mentally retarded and unlikely to have children, but some for unknown reasons are of normal intelligence. It now seems, however, that women with undiagnosed PKU yet of normal intelligence are in danger of producing mentally retarded children. The reason for this seems to be that the high blood levels of phenylalanine in these women can damage the foetus (Perry et al., New Engl. J. Med., 289, $395 ; 1973$ ).

Phenylketonuria is a hereditary disease which occurs in one in 20,000 births and is caused by lack of the enzyme phenylalanine hydroxylase which converts phenylalanine to tyro- sine. In Britain all infants are screened for PKU in the first few weeks of life either by a blood test-which is more reliable-or by a urine test based on detection of the excess phenylpyruvic acid produced as a result of the excess phenylalanine. Untreated, PKU leads to mental retardation, behavioural disorders with psychotic features and fits.

This new work, carried out in British Columbia, arose from an investigation of a patient who had been previously unsuccessfully treated with electroconvulsive therapy (ECT) and drugs for psychotic episodes but was finally referred for biochemical investigation at the age of 46 , possibly as a result of the low IQs-25, 62, 79 and 59of her four children. She was found to excrete large amounts of phenylpyruvic acid, have a very high blood level of phenylalanine and therefore had PKU. Her IQ was 83 .

Two of her three sisters, who were of normal intelligence, also had high phenylalanine levels, and all their children were slow learners at school, although their IQs were not recorded. The third sister had normal levels and children with good school records. The only brother, unmarried, again had high phenylalanine levels, although he was a university graduate. The patient's parents were found to be heterozygote for PKU, as were her children and her siblings.

The authors have reviewed all welldocumented similar cases occurring in 1972, and find that they support the picture of high maternal phenylalanine levels being associated with mental retardation in their children. The higher the phenylalanine levels the more severe the retardation is likely to be.

Ideally, of course, all cases of PKU should be identified by screening in the first or second week of life, but this work shows that possibly this should be repeated on all pregnant women. Further work may show that reducing the phenylalanine levels in the blood by a suitable diet may prevent damage to the foetus. Another moral to this tale is that all patients admitted to mental hospitals ought to undergo biochemical investigation in case there is a recognizable basis for their mental disturbance, otherwise patients may in some cases receive inappropriate treatment.

\section{SLIME MOULDS mRMA Metabolism}

from a Correspondent

THE cellular slime mould Dictyostelium discoideum has long been championed as an ideal model system for the study of morphogenesis and differentiation in metazoan organisms Two papers by Firtel and colleagues in the latest issue of the Journal of Molecular Biology $(79,295$ and 315 ; 1973) suggest, however, that, apart from assisting in these more exalted aims, the slime mould also has a good deal to offer in the way of information about a more basic cellular activity, namely, the synthesis and pro-

\title{
13S DNA in E. coli Ribosome Preparations
}

IN citing DNA as a component of Escherichia coli ribosomal preparations Stygall and Hindley (in Nature New Biology next Wednesday, September 19) are anxious to prove that this is not due to contamination by viruses or fragments of the bacterial genome.

The authors discount the possibility that the DNA is viral in origin by treating the DNA-ribosome complex with DNase before phenol extraction. The DNA is degraded and they reason that, had it been part of a virus, its protein coat would have protected it from degradation.

More evidence is presented that the DNA does not originate from the bacterial genome. First, the monodisperse 13S DNA is shown to be bound to a component in the ribosome preparation and not merely to cosediment with it. Second, when the ribosomes are prepared so as to minimize shearing of DNA (and maintain its high molecular weight), the low molecular weight DNAribosome complex can still be isolated. Third, although the base composition of the DNA shows it to be double stranded, the $\mathrm{G}$ plus $\mathrm{C}$ content is $58.2 \%$ compared with $50 \%$ for total $E$. coli DNA.

Dissociation occurs when the DNAcontaining complex is brought from 10 $\mathrm{mM}$ to $0.1 \mathrm{mM} \mathrm{Mg}^{2+}$. This makes it unlikely to be a bacterial relaxation complex which is stable in the absence of $\mathrm{Mg}^{2+}$. DNA-RNA polymerase and DNA-RNA-RNA polymerase complexes, which might otherwise have been considered as the in vivo site of the $13 \mathrm{~S}$ DNA, are similarly excluded.

Dissociation also occurs when the complex is treated with $T_{1}$ RNase. This leads Stygall and Hindley to suggest that the complex might represent mRNAs in the process of transcription with $30 \mathrm{~S}$ subunits already associated. It would be thought, however, that after attachment of the $30 \mathrm{~S}$ subunit, the $50 \mathrm{~S}$ subunit would combine to form the initiation complex, but although DNA is found in ribosomal preparations it is not found in the $70 \mathrm{~S}$ fraction. The authors' apparent difficulty in suggesting any explanation for this unexpected observation may be due to uncertainty as to the exact binding site of the DNA in their ribosomal preparations. 